LAWRENCE LIVERMORE N A TION AL LABORATORY

\section{Optical Properties of CdSe Nanoparticle Assemblies}

F. R. Leon, N. Zaitseva, D. Gerion, T. Huser, D. M. Krol

November 25, 2003

2003 MRS Fall Meeting

Boston, MA, United States

December 1, 2003 through December 5, 2003 
This document was prepared as an account of work sponsored by an agency of the United States Government. Neither the United States Government nor the University of California nor any of their employees, makes any warranty, express or implied, or assumes any legal liability or responsibility for the accuracy, completeness, or usefulness of any information, apparatus, product, or process disclosed, or represents that its use would not infringe privately owned rights. Reference herein to any specific commercial product, process, or service by trade name, trademark, manufacturer, or otherwise, does not necessarily constitute or imply its endorsement, recommendation, or favoring by the United States Government or the University of California. The views and opinions of authors expressed herein do not necessarily state or reflect those of the United States Government or the University of California, and shall not be used for advertising or product endorsement purposes. 


\title{
Optical Properties of CdSe Nanoparticle Assemblies
}

\author{
F. Rafael Leon ${ }^{1,2}$, Natalia Zaitseva ${ }^{1}$, Daniele Gerion ${ }^{1}$, Thomas Huser $^{1}$, Denise Krol $^{1,2}$ \\ ${ }^{1}$ Lawrence Livermore National Laboratory, \\ 7000 East Avenue \\ Livermore, CA 94550, U.S.A. \\ ${ }^{2}$ University of California at Davis \\ 3001 EU III, One Shields Avenue \\ Davis, CA 95616, U.S.A.
}

\begin{abstract}
We report on three-dimensional fluorescence imaging of micron-size faceted crystals precipitated from solutions of CdSe nanocrystals. Such crystals have previously been suggested to be superlattices of CdSe quantum dots [1,2]. Possible applications for these materials include their use in optical and optoelectronic devices. The micron-size crystals were grown by slow evaporation from toluene solutions of CdSe nanocrystals in the range of 3-6 nm, produced by traditional wet-chemistry techniques. By using a confocal microscope with laser illumination, three-dimensional raster-scanning and synchronized hyper-spectral detection, we have generated spatial profiles of the fluorescence emission intensity and spectrum. The fluorescence data of the micro-crystals were compared with spectra of individual nanocrystals obtained from the same solution. The results do not support the assertion that these microcrystals consist of CdSe superlattices.
\end{abstract}

\section{INTRODUCTION}

Assemblies of CdSe nanocrystals (quantum dots) have potential applications in new optical, electronic and optoelectronic devices. The multipole interaction between neighboring dots suggests that they may prove useful in computational and data storage devices, and their tunable bandgap could allow for the construction of photodiodes and light-emitting diodes in the visible range [1]. The fluorescence emission wavelengths of the quantum dots are tunable by varying their size.

Because the optical properties of the CdSe semiconductor nanoparticles strongly depend on their size, surface structure, and interactions with their environment, it is important to assess how they behave when closely-packed into micrometer-scale assemblies. It has recently been suggested [2] that microcrystals grown from solutions of CdSe nanocrystals consist of crystalline superlattices of CdSe quantum dots. For individual quantum dots to condense into an ordered lattice they must be similar enough to bond together into a regular pattern. For this reason, it was speculated that an ordered superlattice of nanocrystals would consist of similar nanocrystals and thus be more homogeneous than the precursor solution, which typically contains a distribution of nanocrystals of different sizes.

In the present study we have used scanning confocal fluorescence microscopy to characterize micron-size crystals grown from solutions of CdSe nanocrystals. The fluorescence spectra of these crystals are compared to those of individual nanocrystals obtained from the same solution in order to determine whether the fluorescence of the crystals is consistent with an 
ordered superlattice of CdSe nanocrystals, and if so, how uniform the nanocrystal size distribution is inside the micro-crystal.

\section{EXPERIMENTAL DETAILS}

\section{Sample preparation}

Core CdSe nanocrystals were prepared by standard air-free techniques, using TOPO (trioctylphosphine oxide) as a solvent-surfactant and a mixture of $\mathrm{Cd}\left(\mathrm{CH}_{3}\right)_{2}$ and Se as a high temperature precursor $[1,3,4]$. Please note that the quantum dots used for this study consisted of just the TOPO-terminated $\mathrm{CdSe}$ core particle, not the much brighter $\mathrm{CdSe} / \mathrm{CdS}$ system that is often used in biological applications [5]. The nanocrystals were washed by $\mathrm{MeOH}$ three times, dried under nitrogen and dissolved in toluene. The solutions contained quantum dots with a size range of 3-6 nm, as determined by TEM. Initial diluted solutions were left to concentrate by slow evaporation of toluene until they reached saturation to form microscopic faceted crystals. At this point, a drop of solution was placed onto a microscope slide and covered by a Petri dish. Faceted microscopic crystals grew in the drop by slow evaporation of toluene. After the solution dried out completely, crystals were observed under the microscope.

\section{Fluorescence experiments}

For the fluorescence experiments both micro-crystals and individual quantum dots were measured. For the measurements of the micro-crystals the crystals were simply placed onto a glass microscope slide.

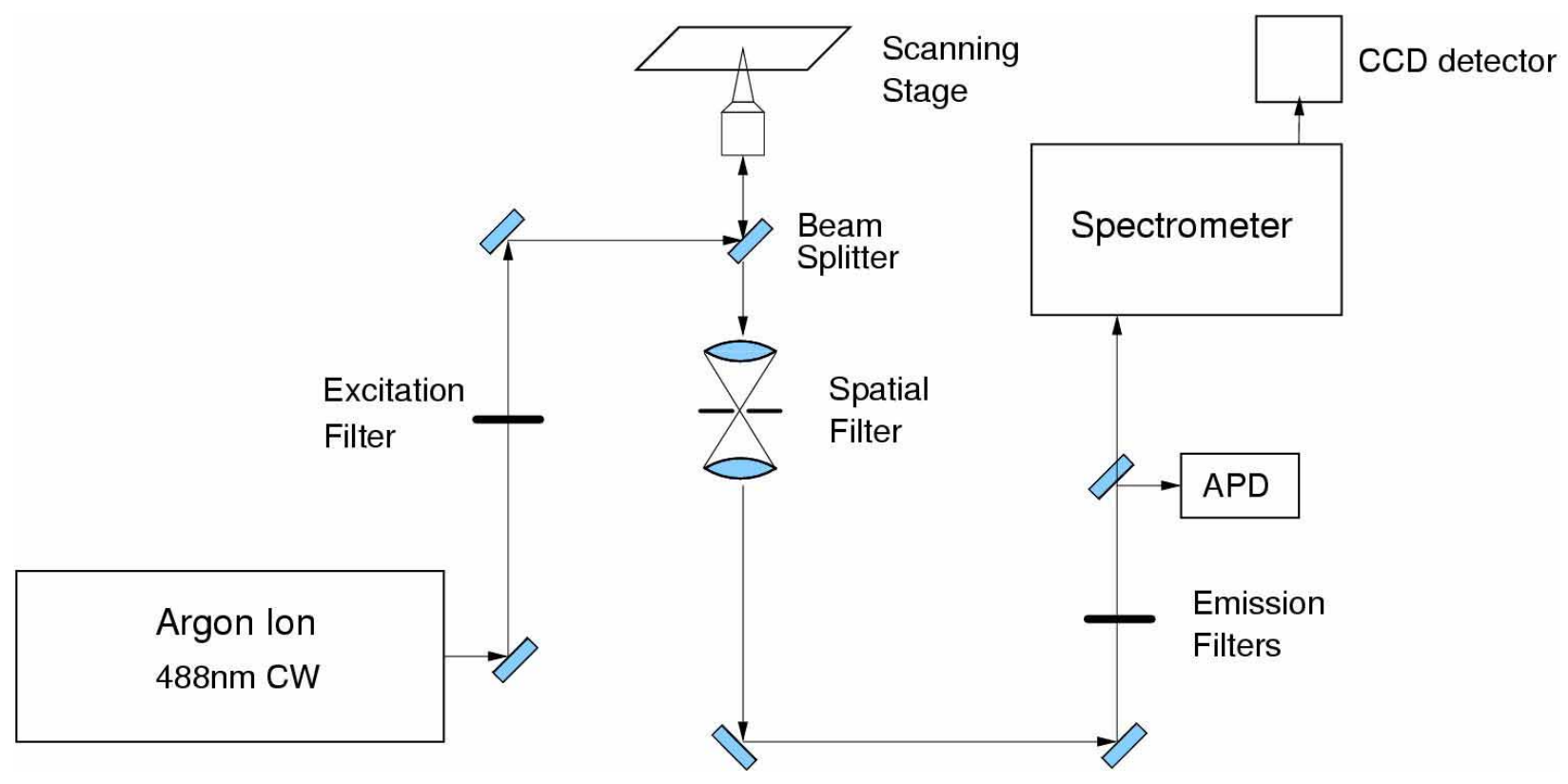

Figure 1: Schematic diagram of the confocal microscope setup used to obtain fluorescence spectra from single microcrystals and individual CdSe quantum dots. 
Figure 1 shows a schematic diagram of the confocal set-up used in our experiments. A collimated Ar+ 488nm excitation laser was filtered using a 488NB3 excitation filter (Omega Optical) and focused through an Olympus 100x 0.90NA air objective to a focal volume on the order of one cubic micron. The microcrystals were then raster-scanned through this focus using a Polytec PI P-500 series piezoelectric stage. Typical scan ranges of $100 \mu \mathrm{m}$ by $100 \mu \mathrm{m}$ and step sizes of $1 \mu \mathrm{m}$ were used. The emitted fluorescence was epi-detected using the same microscope objective. After passing through a spatial filter, this light was chromatically filtered using a 498 AELP long-pass filter (Omega Optical) and BG500 glass filter (Schott) and then sent into an Oriel MS257 spectrometer equipped with a Roper Spec-10:100B CCD multichannel detector. The spectrometer grating had a groove spacing of 300 lines $/ \mathrm{mm}$. Synchronized spectral acquisition was used to acquire spectra every micron during the scanning in order to develop full spatial fluorescence profiles of the crystals. The acquisition time was $50 \mathrm{~ms}$ per pixel, and a $100 X 100$ micron scan with 10,000 pixels had a total acquisition time of 500 seconds.

For the single quantum dot experiments the precursor solutions of nanocrystals were diluted and spin-coated onto glass coverslips in order to spatially isolate single nanocrystals. The coverslips were then excited using the same laser with $8 \mu \mathrm{W}$ of power. The confocal microscope used was also similar, although a Zeiss 1.4NA 100x oil objective was used. Fluorescence intensity detection was performed using an EG\&G SPCM avalanche photodiode. The acquisition time was $1 \mathrm{~ms}$ per 1-micron square pixel. Once located, spectra were taken of single isolated nanocrystals, and these spectra were then compared to the spectra taken of the nanocrystal assemblies.

\section{RESULTS AND DISCUSSION}

Fluorescence spectra were obtained from several select places on each microcrystal. These locations are indicated in figure $2 \mathrm{a}$, and the corresponding spectra are displayed in figure $2 \mathrm{~b}$. Notice that there is negligible variation in spectral profile or intensity from one place to another, even for the spectrum taken from point 2 , i.e. outside the microcrystal. The differences in intensity are due to slight variations in focusing and corresponding confocal collection efficiency. The profile of each spectrum is nearly the same, with a FWHM of approximately $70 \mathrm{~nm}$.
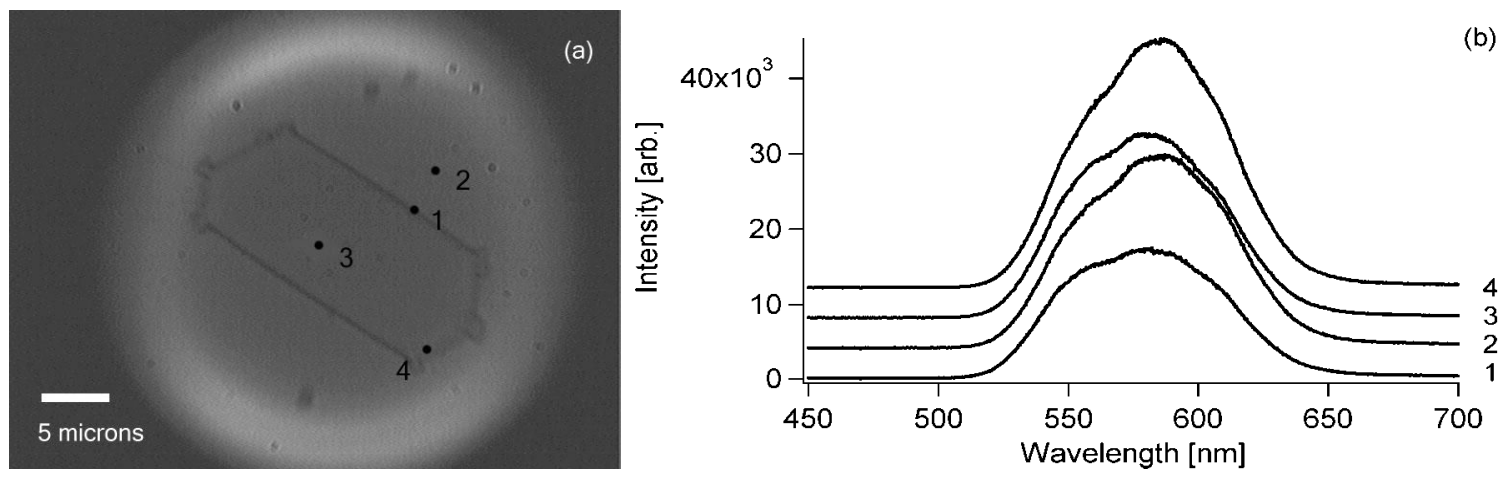

Figure 2. Fluorescence spectra (b) taken from the points on the microcrystal indicated in the white light image (a). The spectra are vertically offset for clarity. 
The only notable difference in these spectra is a slight shift in the overall spectral mean, which is due to slight changes in the peak emission wavelength. The spectrum covers the range from about $520 \mathrm{~nm}$ to $650 \mathrm{~nm}$ with a slight tail to redder wavelengths.

Because of their broad spectral distribution, it was assumed that these spectra resemble ensemble measurements of many quantum dots. In order to confirm this, spectra were taken of single CdSe quantum dots isolated from the same solution from which the microcrystals were grown. By diluting the solutions and dispersing the dots onto a glass coverslip, the nanometersized dots can be spatially separated to a point, where the average distance between adjacent dots is greater than the diameter of the diffraction-limited microscope focus. Subsequently, individual dots can be isolated for spectroscopy. A 10X10-micron scan of several dots is displayed in figure $3 \mathrm{a}$. Some of the nanocrystals luminesce more brightly than others. The brightest spots in the image are the result of clusters of several quantum dots. Emission spectra of these clusters have multiple peaks and indicate the presence of multiple dots. The spectra of several single dots and a representative spectrum of an assembled microcrystal are shown in figure $3 \mathrm{~b}$. The spectra of single CdSe quantum dots have a characteristic spectral width on the order of $20 \mathrm{~nm}$. The difference in emission peak wavelengths between the different dots is due to the different sizes of the dots. Note that the spectra of the individual dots fall under the spectral envelope of the micro-crystal fluorescence, suggesting that the fluorescence of the crystal does not originate from a narrow size distribution of quantum dots.
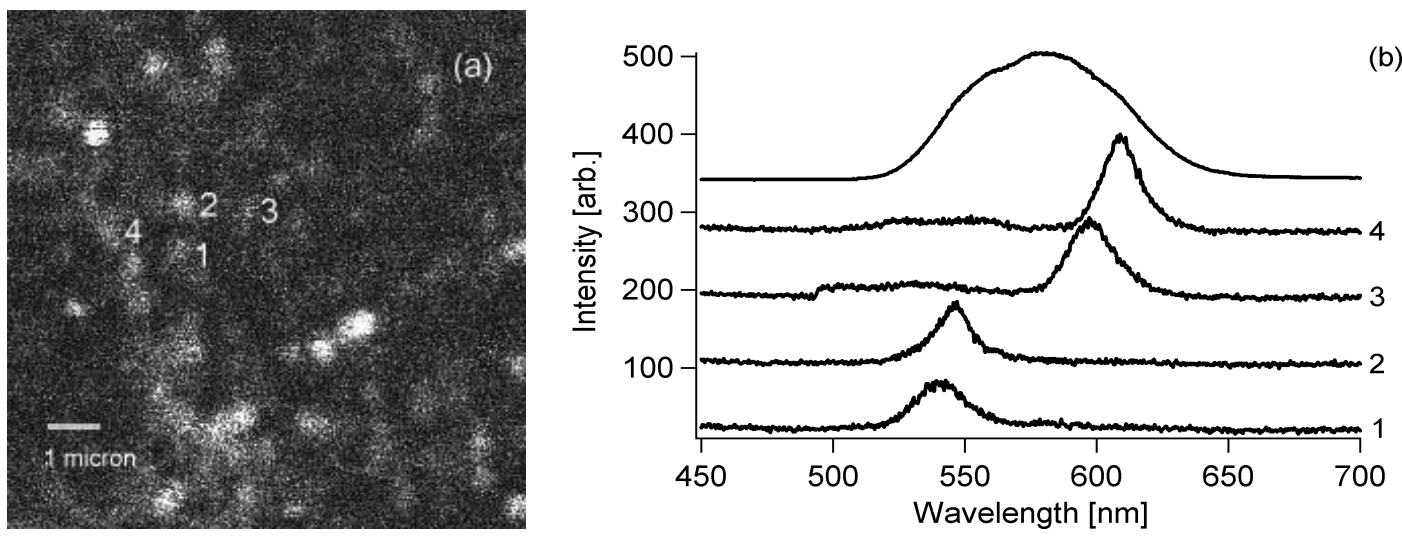

Figure 3. (a) A scanned fluorescence micrograph showing isolated nanocrystals and (b) spectra of four single dots (curves 1-4, taken from positions 1-4 in figure (a)) together with a representative spectrum of an assembled microcrystal (top curve).

As shown in figure $2 \mathrm{~b}$, the emitted fluorescence spectra and intensities obtained from the microcrystal sample vary little with respect to position across the microcrystal surface. In order to verify this fact, a fluorescence intensity scan was performed on several microcrystals. The results of a typical scan are shown in figure $4 \mathrm{a}$. The measured intensity variation has such low contrast that the microcrystal is not discernible from the surrounding background. A white outline was added to the image to indicate the position of the microcrystal. This position was determined by correlating the scan with white-light images. The observed uniform intensity indicates that the microcrystals were covered and surrounded by fluorescent quantum dots. In order to separate the microcrystals from the unassembled fluorescent contaminants, microcrystals bound to the glass surface were rinsed with pure toluene. Similar scans were performed on washed crystals, and the results of such a scan are shown in figure 4b. The crystal 
is in the top right of the picture, and fluorescence is only observed from the meniscus region that forms during the drying process and from several remaining spots of powder on its surface. As the toluene washed over the microcrystal, it dissolved the nanocrystal powder. As the toluene dried, a small number of solvated nanocrystals precipitated within the meniscus at the edge of the microcrystal. The microcrystal is 2 microns thick and its top surface is therefore slightly above the plane of the microscope slide. Nanocrystals also collected in small drops on the surface of the microcrystal and the surrounding glass slide. The collections of nanocrystals on the microscope slide in the bottom left of the figure are slightly out of focus, and therefore less bright.

The fact that the washed microcrystals were found to not fluoresce led us to believe that they were not ordered arrays of nanocrystals. Further experiments using TEM and XRD are under way to precisely determine the nature and structure of these crystals.
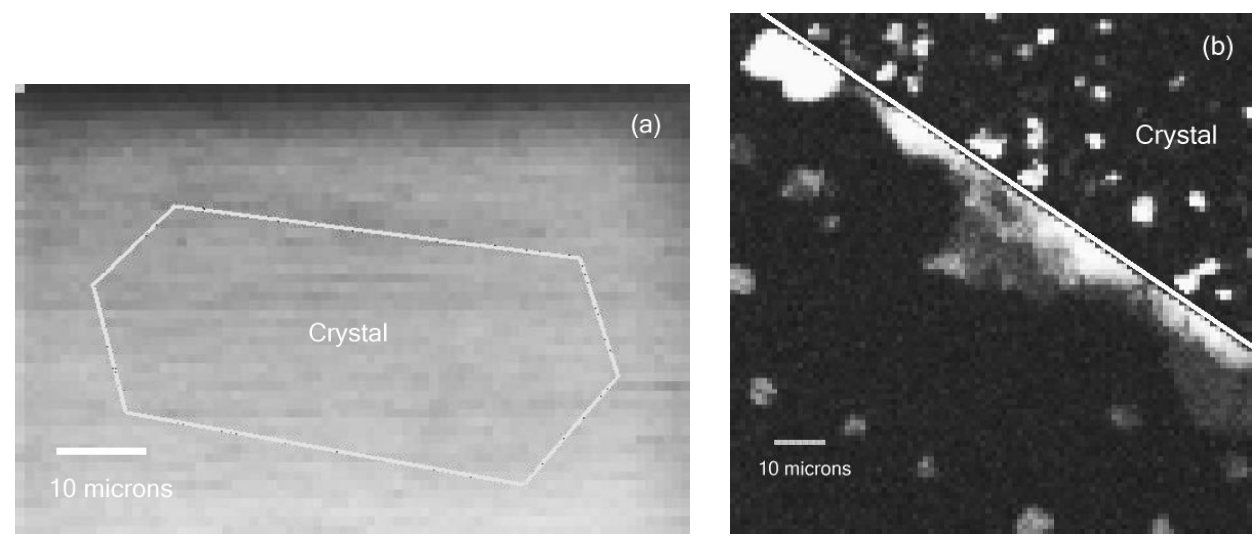

Figure 4. Fluorescence intensity scans of (a) unwashed and (b) washed microcrystals. The white outlines were drawn in to indicate the outline of the crystals as determined from white-light microscopy.

\section{ACKNOWLEDGMENTS}

This work was performed under the auspices of the U.S. Department of Energy, National Nuclear Security Administration by the University of California, Lawrence Livermore National Laboratory under contract no. W-7405-Eng-48. The authors would also like to thank the SEGRF student fellowship program at LLNL for supporting this work.

\section{REFERENCES}

1. C. B. Murray, C. R. Kagan, and M. G. Bawendi, Annual Review of Materials Science, 30, 545-610 (2000).

2. A. L. Rogach, D. V. Talapin, E. V. Shevchenko, A. Kornowski, M. Haase, H. Weller, Adv. Func. Mat., 12 (10), 653-664 (2002).

3. L. Qu and X. Peng, J. Am. Chem. Soc., 124 (9), 2049-2055 (2002).

4. Peng, X. G., Schlamp, M. C., Kadavanich, A. V., Alivisatos, A. P., J. Am. Chem. Soc., 119, 7019-7029 (1997). 
5. X. Michalet, F. Pinaud, T. D. Lacoste, M. Dahan, M. P. Bruchez, A. P. Alivisatos, S. Weiss, Single Molecules, 2 (4), 261-276 (2001). 